\title{
Influence of the gravity on the discharge of a silo
}

\author{
S. Dorbolo, \\ L. Maquet, M. Brandenbourger, \\ F. Ludewig, G. Lumay, \\ H. Caps, N. Vandewalle, \\ S. Rondia ${ }^{\dagger}$, M. Mélard ${ }^{\dagger}$, \\ J. van Loon ${ }^{\ddagger}$, A. Dowson ${ }^{\ddagger}$, \\ and S. Vincent-Bonnieu ${ }^{\ddagger}$
}

Received: date / Accepted: date

\begin{abstract}
We performed a series of experiments to investigate the flow of an assembly of non-cohesive spherical grains in both high and low gravity conditions (i.e. above and under the Earth's gravity). In high gravity conditions, we studied the flow of glass beads out of a cylindrical silo and the flow of metallic beads out of a vertical Hele-Shaw rectangular silo. Both silos were loaded in one of the gondolas of the Large Diameter Centrifuge facility (located at ESTEC) in which an apparent gravity up to 20 times the Earth's gravity can be established. To simulate low gravity conditions, we submitted a horizontal monolayer of metallic beads to the centrifuge force of a small rotation device (located at University of Liege). The influences of both gravity and aperture size on the mass flow were analysed in these various conditions. For the three systems (cylindrical silo, the Hele-shaw silo and the monolayer of beads), we demonstrated that (i) the square root scaling of the gravity found by Beverloo is relevant and (ii) the critical aperture size below which the flow is jammed does not significantly increase with the apparent gravity. Moreover, we studied in more details the Hele-Shaw silo in high gravity because this configuration allowed to determine local properties of the flow at the level of the aperture. We measured the velocity profiles and the packing fraction profiles for the various aperture sizes and apparent high gravities. We demonstrate the existence of a slip length for the flow at the level of the aperture. This later fact seems to result from the geometrical configuration of the silo.
\end{abstract}

Keywords granular flow · gravity

$\overline{\text { GRASP, Physics Department }}$

$\dagger$ Faculty of Sciences

University of Liège, B-4000 Liège, Belgium.

$\ddagger$ ESA / ESTEC, Noordwijk, The Netherlands 


\section{Introduction}

When one considers a non-cohesive granular packing, one considers a complex network of grains in contact submitted to a homogeneous field, the gravity. Indeed, each grain is submitted to three forces: their weight, the normal forces resulting from the contact with their neighbours and the tangential friction forces also due to the contacts. The granular material properties like the packing fraction or the flowability, are related to the complex force network resulting from the numerous contacts between the grains. As the forces (the weight and indirectly the contact and the friction forces) result from the presence of the gravity, one could wonder how the gravity modifies the granular properties such as the shape of the heap, the dynamics of the avalanches, the packing fraction or the flowability. These properties are relevant regarding, for example, the exploration of asteroids or planets. Indeed, these celestial objects are covered by dust, sand and rocks and are more often then not characterized by a gravity smaller than the Earth's gravity. In Ref.[1], Scheeres et al. summarized how the cohesion is affected in reduced gravity conditions. However, very few experimental works can be found about the influence of the gravity on granular material properties.

Experimentally, to change the apparent gravity without traveling to another planet, three strategies can be found in the literature. Either, the apparent gravity $g^{\star}$ is increased using a Large Diameter Centrifuge (LDC), see Fig. 1a-b, or $g^{\star}$ is decreased using parabolic flights, or finally, $g^{\star}$ is decreased by rotating an horizontal monolayer of beads, see Fig. 2a-b.

Granular material confined in rotating tumbler has been studied under various apparent gravity conditions [2-4]. The idea was to determine how avalanches are influenced by the gravity and to determine the role of the gravity in geomorphology of planets. The basic physical properties that characterise the avalanches dynamics were measured as a function of the apparent gravity, namely the repose angles (static and dynamic) and the flow layer thickness. In Ref.[3], the static and dynamic angles of repose are shown to be independent of the apparent gravity in the range $g<g^{\star}<25 \mathrm{~g}$. The Authors also concluded that the flow layer thickness only depends on the particle size and that the apparent gravity only influences the shear rate [2]. These results are questioned in Ref.[4]. In this paper, the Authors studied tumblers in parabolic flight. In the range $g^{\star}=0.1$ and $0.38 \mathrm{~g}$, they concluded that the static angle of repose decreases when the apparent gravity increases while the dynamics angle of repose increases with the apparent gravity. The Authors also evidenced the role of the packing fraction (ratio between the volume of the grains and the volume occupied by the packing) of the granular material that flows. Recently, we propose an alternative way to tune the apparent gravity below $g$. The method only applies to a planar monolayer of grains. A horizontal monolayer of millimetric beads was fixed at the extremity of an arm that rotated horizontally. The in-plane apparent acceleration depends on the rotation speed. We measured that the density of the packing fraction (at rest) 


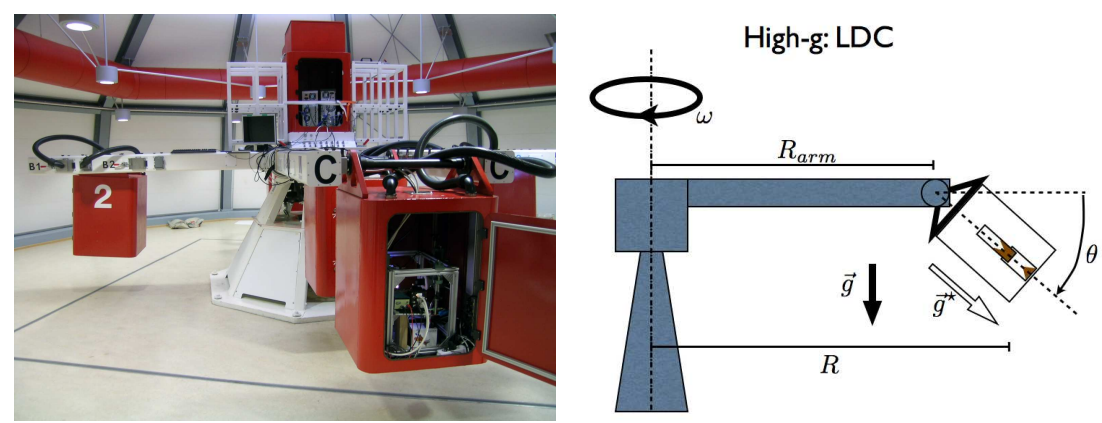

Fig. 1 2D and 3D high gravity (a) Picture of the LDC ESA facility located at ESTEC (Credits:ESA). See movie [6]. (b) Schematic representation of the gondola in rotation.

varies from 0.80 to 0.84 while the apparent gravity $g^{\star}$ is increased from $0.05 \mathrm{~g}$ to $0.2 \mathrm{~g}$. The packing fraction saturates above $g^{\star}=0.5 \mathrm{~g}[5]$.

In the present paper, we investigate the influence of the gravity on a very classical granular material flow: the discharge of a silo. Silos remain the main way to stock granular material and are consequently found in a wide range of industrial processes: pharmaceutical engineering, agriculture, powder industry... The flow out of the silo and the jamming of the flow have been intensively studied. The remarkable constancy of the outflow, whatever the height reached by the granular material in the silo is used to measure time with hourglass since the 10th century. However, the flow out of a silo was first systematically studied by a Swiss apiculturist F. Huber-Burnand [7] ${ }^{1}$. Another property of the flow is that, contrary to a fluid, below a given size of the aperture, the outflow is jammed. Both properties were rationalised by Beverloo through a phenomenological law which links the outflow $Q$ (number of grains per second) to the grain size $d$ and to the aperture size $D[8]$ :

$$
Q=A \rho_{B} \sqrt{g}(D-k d)^{2.5}
$$

where $A$ depends on the geometry and on the friction, $\rho_{B}$ is packing fraction of the bulk, $k$ is a constant depending on the geometry and on the friction between beads (=1.4 in ref.[8]) and $g$ the gravity acceleration. To explain the constancy of the outflow, the role of the force network, especially the redirection of force towards the wall were evidenced [9-11]. The pressure on the wall and on the bottom was revisited more recently by experiments on $2 \mathrm{D}$ flow driven by a conveyor belt [12-14]. In these experiments, the speed of the grains can be varied by the speed of the belt. This is, in some extensions, the idea of the high gravity experiments: to increase uniformly the force re for the grain motion. However, Géminard and coworkers concluded after a very detailed experiment: "the physical origin of the Beverloo law remains unclear and still requires additional efforts to be elucidated" [15]. As clearly stated

\footnotetext{
1 Note: François Huber-Burnand (1750-1831) was blind since he was 15. He was helped by his domestic François Burnens during his experiments in Yverdon-les-Bains (Switzerland).
} 


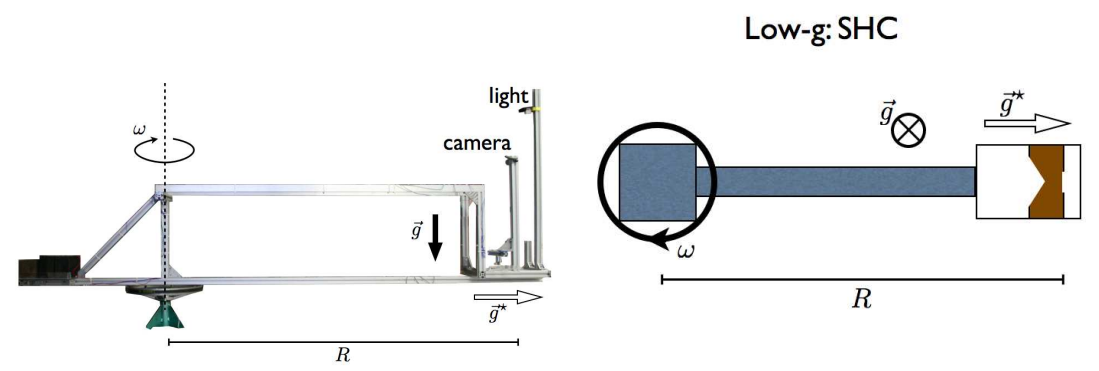

Fig. 2 2D low gravity (a) Picture of the horizontal rotation silo. (b) Schematic representation of the horizontal Hele-Shaw silo in rotation.

in this paper, the relationship between the flow out of the silo and the force network is still to be debated.

The existence of a critical size for the aperture $D_{\min }$ below which the flow is jammed appears to be only natural. Obviously, the value of the critical size depends on the relative size of the grains compared to the aperture size. Moreover, close to this critical value $D_{\min }$, the Beverloo's law has to be modified in order to fit experimental data obtained for small apertures compared to the grain size $[17,18]$. This law was also confronted to different situations: when grains are cohesive using a magnetic field [19] or using a electric field [20], or even when the grains are deformable [21]. It is also remarkable that local vibrations close to the aperture [22] or the presence of an obstacle [23] decreases the critical size of the aperture. This approach evidenced that the flow is driven by the local speed and volume fraction of the granular material close to the orifice. A fine analysis can be found in the Ref. [24] and is also supported by the arch formation analysis $[25,26]$.

In this paper, we propose (i) to experimentally test the scaling of Beverloo's law regarding to the gravity acceleration and (ii) to experimentally observe whether $D_{\min }$ is modified by the apparent gravity. It is clear that the maximum speed of the grains at the aperture of the silo cannot but scale with the square root of the gravity. However, it is not immediate that the flow will follow the same scaling as it depends on the velocity profile and the packing profile of the flow.

For this purpose, we performed experiments at the Large Diameter Centrifuge facility at ESTEC that allows to reach local apparent gravity acceleration up to 20 times the Earth's gravity. Two experimental set-up were designed for this purpose: (i) Cylindrical silos that contained sand (referenced as 3D in the following) and (ii) Hele-Shaw silos that contained a monolayer of spherical beads (referenced as $2 \mathrm{D}$ in the following). The advantage of the $2 \mathrm{D}$ configuration is that individual grains can be tracked close to the aperture. Consequently, we are able to measure local properties like velocity and packing fraction profiles. The set-up were loaded in one of the gondola of the Large Diameter Centrifuge (LDC) located at ESTEC (ESA, Noordwijk, The Nether- 
(a)

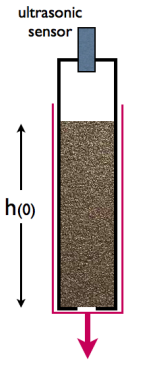

$\mathrm{t}=0$

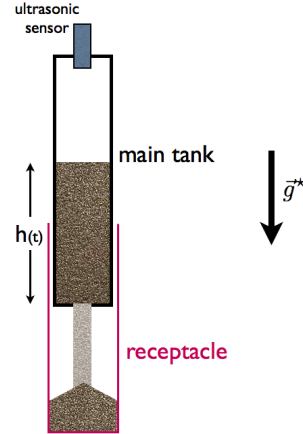

$\mathrm{t}>0$

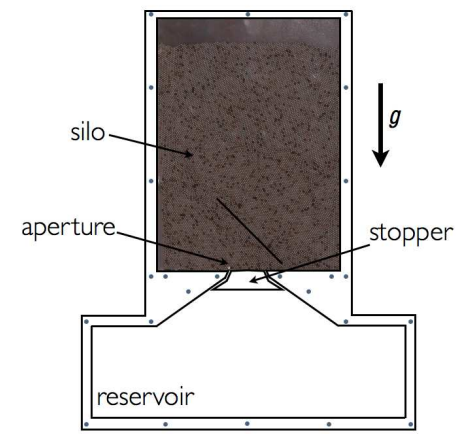

(b)

Fig. 3 3D (a) Four cylinders were aligned in the gondola in order to test 4 aperture sizes in the same time. On the Figure, one of these cylinders is sketched. They are equipped with ultrasonic sensor at the top of each cylinder. These sensors are able to detect the air-grains interface. The mobile part of the silo is constituted by the cylinders (in red). Their motion towards the bottom of the gondola liberates the grains that can flow through the orifice. The motion is driven by a step motor. 2D (b) Sketch of the experimental set-up. The silo dimensions are $30 \mathrm{~cm}$ of height, $20 \mathrm{~cm}$ of width. The separation between both vertical plates of confinement is $2.1 \mathrm{~mm}$ that allows the $2 \mathrm{~mm}$ stainless steel beads to freely move. The aperture size can be changed. A stopper is remotely commanded and releases the beads towards the reservoir. A high speed camera recorded the motion of the beads close to the aperture.

lands). Apparent gravities up to 20 times the Earth's gravity can be applied to the experimental set-up. We chose to focus on aperture size not too close from the critical aperture because, as we will detail further, the vibrations of the centrifuge may affect the flow. Moreover, we analysed the local behaviour of the granular material close to the aperture and this, for different values of the apparent gravity.

Our observations experimentally validate the gravity scaling found by Beverloo for the flow out of the silo. And, regarding our data, we can conclude that the apparent gravity does not significantly influence the minimum size of the aperture required to avoid the flow to be jammed. Finally, the study of the local parameters (velocity and packing fraction profiles) brings to light the existence of a slip length of the outflow that is independent of the apparent gravity but only on the geometrical configuration of the silo. To complete the experimental investigations, we also performed discharge of monolayer silos in low gravity conditions using a similar experimental set-up described in ref.[5]. The results confirm the high gravity outcomes. 


\section{Experimental details}

2.1 High gravity: 3D and 2D set-up

The experimental set-up was loaded in one of the gondolas of the Large Diameter Centrifuge (LDC). The gondola is attached at the extremity of one of the four arms of the centrifuge, namely at $4 \mathrm{~m}$ from the center of rotation of the centrifuge. During the rotation, the gondola inclines with an angle $\theta\left(\theta=90^{\circ}\right.$ when $\omega=0$ ) in order to keep the vertical direction towards the bottom of the gondola, see Fig.1b for the geometry of the system. The angle $\theta$ is given by the relation

$$
\tan (\theta)=\frac{g}{\omega^{2} R}
$$

where $R$ is the distance from the point of interest to the center of rotation and $\omega$ the angular velocity. The apparent gravity $g^{\star}$ is then found by calculating $R \omega^{2}$. The results are presented as a function of the adimensional gravity $\Gamma=g^{\star} / g$. An apparent gravity up to 20 times the Earth gravity can be obtained at the bottom of the gondola; at this moment, $\theta$ equal $2.86^{\circ}$. Because the artificial gravity depends on $R$, a gradient of gravity exists inside the gondola. The gradient of apparent acceleration along the horizontal direction relative to the gondola is given by $\omega^{2} \cos (\theta)\left(\mathrm{m} \mathrm{s}^{-2}\right)$ which is maximum when the rotation speed is maximum. For example, when $\Gamma=20$ at the bottom of the gondola, the apparent gravity is only $\Gamma=19.6$ at $40 \mathrm{~cm}$ above the bottom (see movie $[6])$.

The experimental procedure decomposed as following. The system is rotated until the apparent gravity $g^{\star}$ is stable. The aperture is then remotely opened and synchronized to the image acquisition. The LDC is finally decelerated, the system is reset.

3D set-up The silo consisted in a vertical cylindrical tank $\left(R_{c}=20 \mathrm{~mm}\right)$. This main cylinder was filled with glass beads ( $d$ between 200 and $400 \mu \mathrm{m}$ ) [27]. At the bottom of that main tank, a hole of diameter $D$ was drilled. The cylinder fitted inside a larger cylinder, the receptacle, which is closed at its bottom end. When the main cylinder is completely inside the larger cylinder, the silo exhaust is blocked by the bottleneck of the receptacle (Fig.3a). The receptacle was entrained down by a remotely commanded step motor. The down motion of the receptacle results in the opening of the aperture of the main cylinder. Four silos were put in parallel in order to measure the outflow $Q$ for four apertures in one run (namely $D=2.7,5.0,7.8$, and $10.35 \mathrm{~mm}$ respectively).

A proximity sensor was placed at the top end of the main tank in order to determine the position $h(t)$ of the free surface of the glass bead packing. As the outflow is constant, the function $h(t)$ can be fitted by a linear law $h(t)=a\left(t_{0}-t\right)$ where $a$ and $t_{0}$ are fitting parameters. The determination of the coefficient $a$ allows to calculate the flow $Q=a \pi R_{c}^{2}$.

2D set-up For the confined configuration, we built a silo in a Hele-shaw cell (see Fig. 3b). The dimension of the silo were $300 \times 200 \times 2.1 \mathrm{~mm}^{3}$. About 18000 stainless steel beads of $d=2 \mathrm{~mm}$ diameter were placed between the 


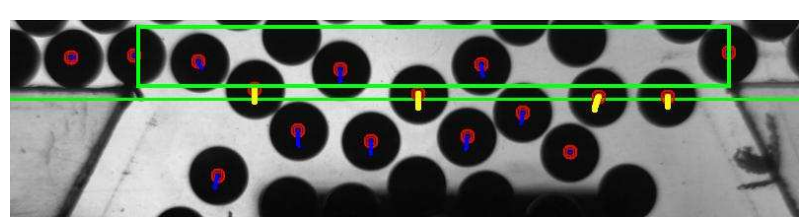

Fig. 4 2D (colour online) Snapshot of the silo discharge $(D=20 \mathrm{~mm}$ and $\Gamma=13)$. The green horizontal line represents the exit line. When the beads cross the line, they are considered as being out of the silo. The red circles represent the center of mass of each beads, the blue lines correspond to the displacement of each bead relatively to the next snapshot (time separated by $250 \mu \mathrm{s}$ ). The measurement box is also represented by a green rectangle. The local packing fraction profile $\phi(x)$ is defined as the proportion of pixels belonging to the beads compared to the total pixels of one column of pixels of the box.

walls of this cell. An adjustable aperture is set at the bottom short side. The aperture size $D$ can be tuned between 10 an $40 \mathrm{~mm}$ (by steps of $5 \mathrm{~mm}$ ). The aperture was blocked using a stopper which was held by a pin. This pin can be remotely retracted using a servo-motor. The motion of the beads close to aperture was recorded using a high-speed camera (N3 IDT-vision) at 4000 fps. The illumination was ensured by a LED spot on the other side of the hele-shaw cell and directed towards the camera so as to visualise the "shadow plays" of the silo discharge. The aperture of the silo and the image acquisition were synchronised using a National Instrument DAQ (NI-6251).

The great advantage of the $2 \mathrm{D}$ set-up is to allow the tracking of individual particles during the discharge. In Fig.4, a typical snapshot of a movie is presented. The grains are flowing out of the silo that has an aperture size $D=20$ $\mathrm{mm}$; the apparent gravity is $\Gamma=13$. The origin of the axis is located at the center of the aperture. The $x$-axis is chosen parallel to the aperture, while the $y$-axis is along the vertical direction (Fig.4). The position of the beads on each image is determined by image analysis. The bead positions are determined by the center of mass (red circles) of the dark circular spots. From one snapshot to the following one, the displacements of the beads are determined by a particle tracking algorithm. The displacement vectors are represented by blue lines. Three physical values are then computed from the position measurements: the mass flow $Q$, the velocity profile $v_{y}(x)$ along the vertical direction and the local area fraction $\phi(x)$.

The flow $Q$ out of the silo is determined by counting the number of beads that cross a virtual horizontal line (so called the exit line) that links both sides of the aperture (horizontal green line in Fig.4). The flow, expressed in number of beads by second, is given by the number of beads crossing the exit line by image divided by the frame rate.

The velocity profile along the $y$ direction is obtained after the determination of the speed of the grains. The method is similar to that found in Ref.[24]. The vertical component of the speed $v_{y}$ is found by projection of the displacement vectors on the $y$-axis. The profile $v_{y}(x)$ is obtained by averaging the vertical speed over 1500 frames of the movie (at $4000 \mathrm{fps}$ ). 
Finally, the local packing fraction is measured. A common box of interest is defined in the snapshots. This box is located just above the aperture (see Fig. 4). The length and the height of the box are given by the size of the aperture and by the bead diameter respectively. For each value $x$ between $x=-D / 2$ and $x=D / 2$, it is possible to calculate the proportion $\phi(x)$ of pixels belonging to the beads compared to the height of the box measured in pixels. The quantity $\phi(x)$ is obtained after averaging the local packing fraction over 1500 frames of the movie (taken at $4000 \mathrm{fps}$ ).

\subsection{Low gravity set-up}

The horizontal silo was made of a glass plate which dimensions was $200 \times 300$ $\mathrm{mm}^{2}$. The same beads as in the Hele-Shaw vertical silo were used. The plate was then horizontally fixed at the extremity of an arm which rotated horizontally with a constant speed. The middle of the silo was located at $R=1.6$ $\mathrm{m}$ from the rotation axis. The Fig. $2 \mathrm{a}$-b shows a picture of the experimental set up. When the system rotates, the beads move away from the center and flow out through an aperture located at the middle of the side that was the furthest from the rotation axis. The acceleration $g^{\star}$ is given by $R \omega^{2}, R$ being the distance between the axis of rotation and the middle of the plate. The minimum apparent gravity $g^{\star}$ must be larger than $0.3 \mathrm{~m} / \mathrm{s}^{2}$ in order to allow the grains to roll.

A gate blocked the aperture and was remotely opened. The flow was then recorded using a SONY HD camera. By image analysis, we measured the flow out of the silo with respect to the apparent gravity and to the aperture size. As the beads are rolling, the situation is pretty different from the high gravity conditions for which the grains are falling. That is the reason why we chose to test in details only one aperture, i.e. $D=8.5 d$ (and partially $D=5 d$ and $12 d)$.

\section{Results}

3.1 High gravity: 3D case

In Fig. 5, typical results from the experiments are presented. In Fig. 5a, the gravity is fixed to $\Gamma=1$. The evolution of the heights $h$ reached by the sand in each cylinder (having different aperture size) is plotted as a function of the time, i.e. the red circles, the blue squares, the red diamonds and the black triangles correspond to an aperture size $D$ of 2.7, 5.0, 7.8 and 10.25 $\mathrm{mm}$ respectively. The origin of time is determined by the opening of the silos. Starting from a height of approximatively $220 \mathrm{~mm}$, the heights decrease with time faster and faster when the diameter of the orifice is larger. In Fig. 5b, the diameter size is remained constant. The different curves correspond to different reduced gravity (see legend). As expected, the height $h$ of the sand decreases faster for large values of $\Gamma$. 


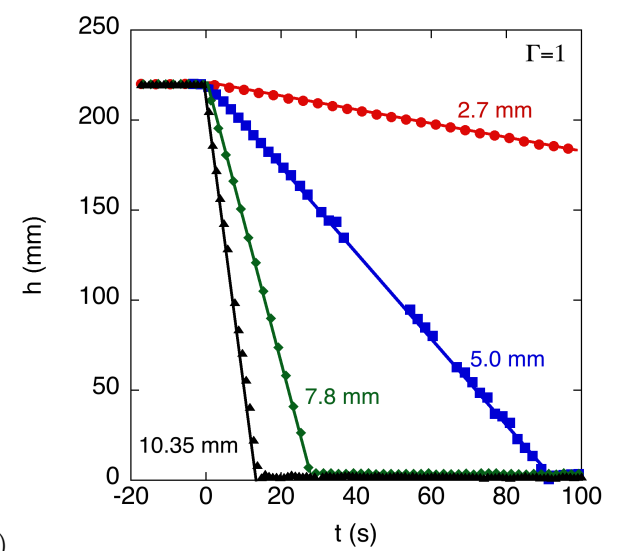

(a)

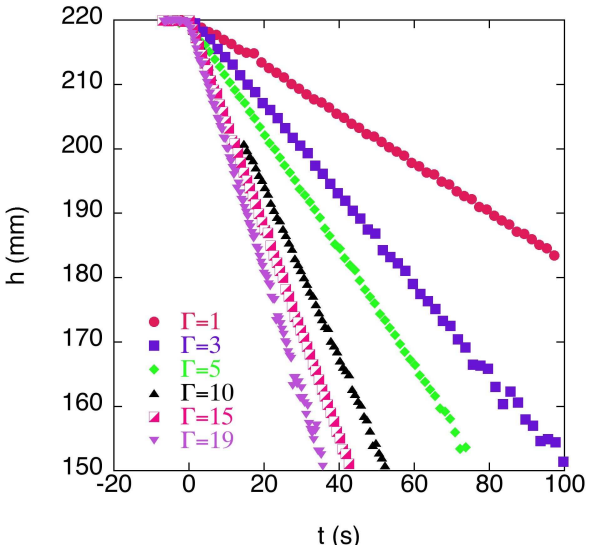

(b)

$t(s)$

Fig. 5 3D Typical results of silo discharge. (a) The gravity is fixed to $\Gamma=1$. The time evolution of the sand height $h$ in the silo is presented for 4 different aperture size $D=2.7$ $\mathrm{mm}$ (red bullets), $5.0 \mathrm{~mm}$ (blue squares), $7.8 \mathrm{~mm}$ (green diamonds), and $10.35 \mathrm{~mm}$ (black triangles). (b) The time evolution of the sand height in the cylinder is presented for an aperture of $2.7 \mathrm{~mm}$. The different curves correspond to different apparent gravities (see legend). In both figures, only a few percentage of the data is presented for clarity.

The flow rates $Q$ has been measured for the four aperture sizes and for 10 different apparent gravities $\Gamma$. In Fig. 6, the flow $Q$ is plotted as a function of the apparent gravity $\Gamma$ in a log-log plot for the four considered apertures. The symbols are the same as in Fig. 6. The lines correspond to fits by a square root law, namely

$$
Q=\alpha(D) \sqrt{\Gamma}
$$

where $\alpha$ depends on the aperture size. A good agreement is found with the square root of the gravity.

The jamming of the flow that occurs when the size of the aperture $D$ is below a critical value $D_{m i n}$, is sensitive to vibrations [22]. In ref.[22], Janda et al. showed that the minimum size $D_{\min }$ decreases linearly with the acceleration 


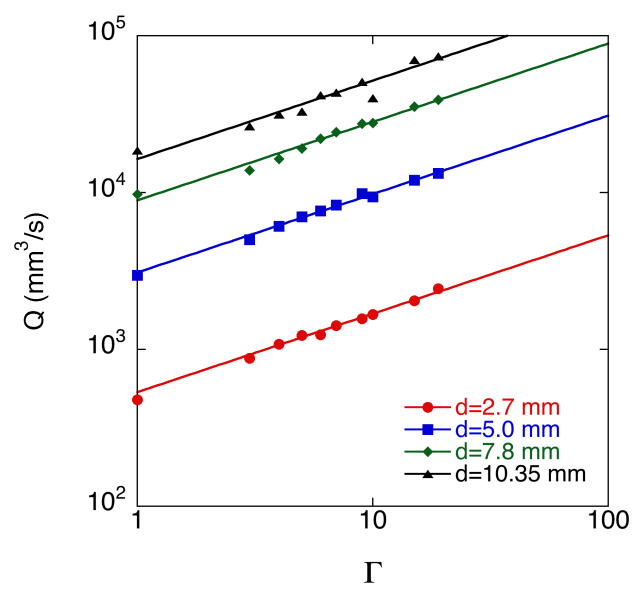

Fig. 6 3D Evolution of the flow out of the four silos which aperture size are $D=2.7$ $\mathrm{mm}$ (red bullets), $5.0 \mathrm{~mm}$ (blue squares), $7.8 \mathrm{~mm}$ (green diamonds), and $10.35 \mathrm{~mm}$ (black triangles). The lines are fits by Eq.(2).

of the vibration (at a fixed frequency $350 \mathrm{~Hz}$ ). Regarding the vibration of the LDC, the measurements [28] reports a maximum acceleration of the vibration of $0.01 \mathrm{~g}$ at $35 \mathrm{~Hz}$. For comparison, as suggested in ref.[22], it is better to evaluate the mean kinetic energy injected by the vibration. At worse, we found that the injected energy by the vibration of the LDC corresponds to the energy injected when the acceleration is $0.1 \mathrm{~g}$ at $350 \mathrm{~Hz}$. According to ref.[22], that means that we have to expect an increase of $D_{\min }$ of $30 \%$. As no jamming has been observed for any effective gravity, even for the smallest aperture, that signifies that the arch creation-destruction dynamics is independent of the gravity or at least, the influence of the gravity is much smaller than the influence of very small vibrations. The force network is affected as the apparent weight of the grains is increased. On the other hand, we might expect that the geometry of the force network remains unchanged.

\subsection{High gravity: 2D case}

To begin with, let us apply the Beverloo's law to the $2 \mathrm{D}$ case. The flow $Q$ out of an orifice is given by the product of the mean speed of the fluid $v_{\text {out }}$ and of the aperture size. As we deal with spherical particles, the flow $Q$ expressed in number of beads passing through the orifice per second is given by

$$
Q=\frac{4 \bar{\phi}}{\pi d^{2}} v_{\text {out }} D
$$

where $\bar{\phi}$ is the mean area fraction and $d$ the diameter of the beads. Classically, the Beverloo's law is deduced from two hypothesis: (i) the flow $Q$ is blocked 


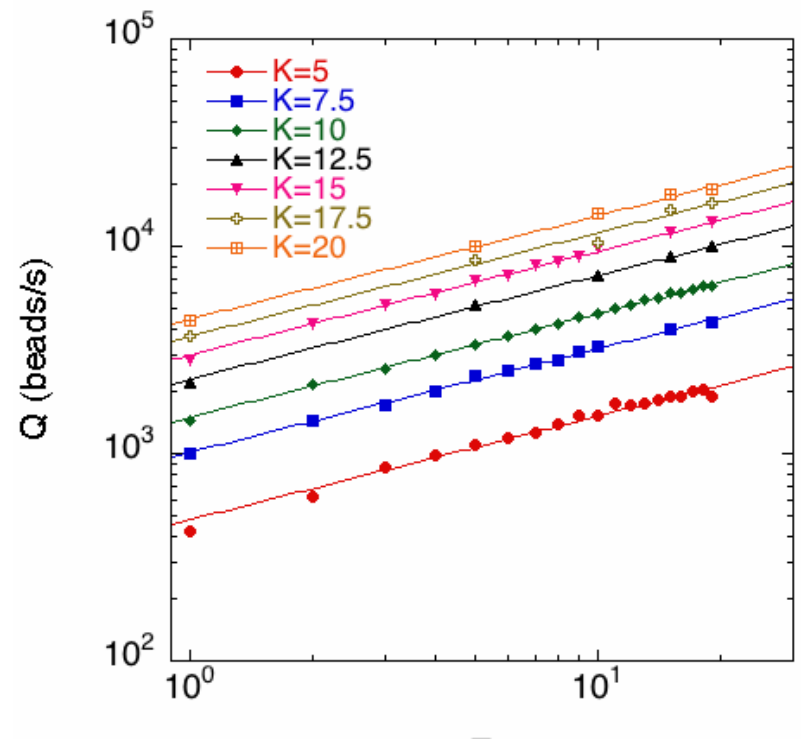

(a)

$\Gamma$

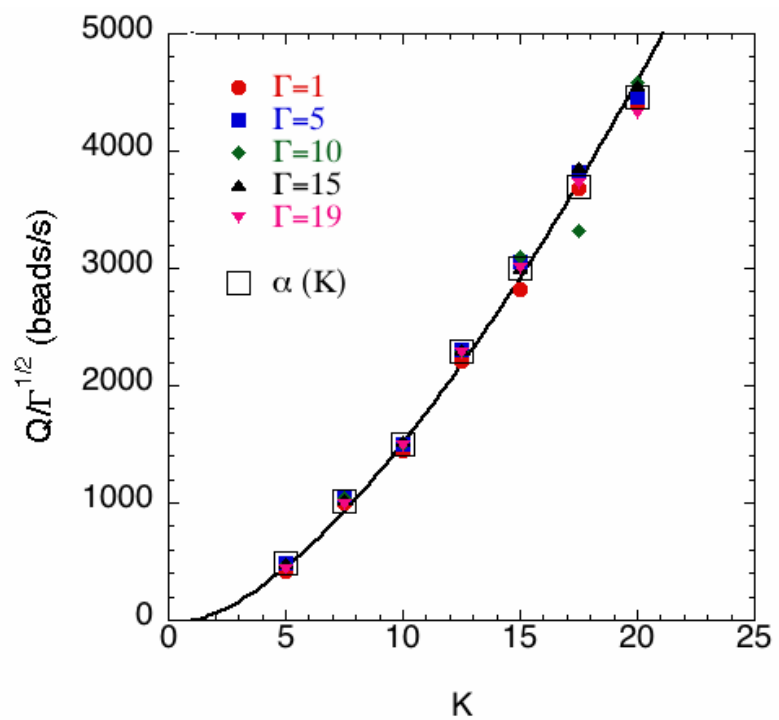

(b)

Fig. 7 2D (a) Flow $Q$ out of the $2 \mathrm{D}$ silo as a function of the apparent reduced gravity $\Gamma$ for different normalized aperture size $K$ (see legend). (b) Flow normalized by the square root of the apparent gravity, i.e. as a function of the aperture size $K$ for different reduced accelerations $\Gamma$. The solid line is a fit by Eq.(4). 
when the aperture is below a threshold given by $k d$ where $k$ is a free parameter and $d$ the bead diameter. In other words, the value $k d$ corresponds to the minimum aperture size $D_{\text {min }}$ below which the flow is jammed. (ii) The grains experience a free fall before passing through the aperture. This hypothesis comes from the idea that the jamming mechanism is due to the formation of a semi-circular arch around the aperture $[25,26]$. When the arch breaks, the grains fall and arrive at the aperture with a speed equivalent to a free fall from a height $\beta D$ where $\beta$ is a geometrical factor. The speed of the grains is then given by $v_{\text {out }}=\sqrt{2 g^{\star} \beta D}$. If one considers that arches are semi-circular, $\beta$ is equal to 0.5 . In the following, $\beta$ is a free parameter. Finally, the flow can be rewritten as

$$
Q=\frac{4 \bar{\phi}}{\pi d^{2}} \sqrt{2 g^{\star} \beta D}\left(D-D_{\text {min }}\right)
$$

where $\bar{\phi}$ is the mean area fraction at the outlet (which depends on the size aperture). The characteristic distance $\beta D$ will be investigated in the next section. Finally, we can define a rectangular area $A_{f}$ upstream the orifice in which the granular material can be considered as fluidized. This area is given by $A_{f}=\beta D^{2}$.

Note that the Eq.(4) is slightly different from the original Beverloo's law (Eq.(1) at 2D, the exponent 2.5 becomes 1.5 see Ref.[24]). Basically, we claim that the fluidized area is a function of $D$ and not of $D-D_{\min }$ when the grains can flow out of the silo, namely when $D>D_{\min }$. Indeed, when considering apertures just below $D_{\text {min }}$, the flow is blocked by a semi-circular (in first approximation) arch of radius $D / 2[26]$. This length is the characteristic size of the fluidized area and not $D-D_{\min }$.

In the following, we present the influence of the reduced apparent gravity $\Gamma$ and of the reduced aperture size $K=D / d$ on the flow $Q$ out of the silo. In addition, we will discuss the influence of $\Gamma$ on the minimum aperture size $D_{\text {min }}$. Afterwards, we will report the local measurement analysis and their relationship with the global flow.

\section{Global flow}

In Fig. 7a, the flow $Q$ (number of beads $n$ per second) is reported as a function of the square root of the normalized apparent gravity $\Gamma$ and this for different reduced aperture sizes $K$ of the silo. The reduced aperture size $K$ has been tuned between 5 and 20. For any aperture, the flow increases with $\Gamma$. The lines correspond to fits by the following law

$$
Q=\alpha(K) \sqrt{\Gamma}
$$

where $\alpha$ is the only fitting parameter. The agreement with the square root law is remarkable. This clearly demonstrates that the discharge of the silo is driven by the free fall of the grains.

Concerning the influence of the apparent gravity on the minimum size of the aperture, we can conclude in a similar way as in the $3 \mathrm{D}$ case. In the $2 \mathrm{D}$ case, we found that the jamming occurs for an aperture size between $D=5$ and $10 \mathrm{~mm}$. The vibration could increase the minimum aperture of $30 \%$. That 
means that the jamming should occur for an aperture size between 8 and 13 $\mathrm{mm}$ for $\Gamma=20$. Actually, the smallest aperture we considered was $10 \mathrm{~mm}$. No jamming effect has been observed. We can conclude that the apparent gravity does not significantly increase the value of $D_{\min }$.

In Fig.7b, we report the flow $Q$ divided by $\sqrt{\Gamma}$ as a function of $K$. We report also the fitted values $\alpha(K)$. The curve is obtained by fitting the following equation

$$
\frac{Q}{\sqrt{\Gamma}}=\xi \sqrt{K}(K-1)
$$

which corresponds to the Beverloo law, i.e. Eq.(4) with $k=1$ (which is the minimum possible value). Comparing Eq.(6) and Eq.(4), one finds that

$$
\xi=\frac{1}{2 g}\left(\frac{\pi \xi \sqrt{d}}{4 \bar{\phi}}\right)^{2} \approx 53.6 s^{-1}
$$

the numerical value results from the fit. If we determine the average packing fraction $\bar{\phi}$ close to the aperture, it is then possible to calculate the value of $\beta$ and consequently, to calculate the fluidized surface $A_{f}$.

\section{Local parameters}

The local parameters, i.e. the vertical velocity profile $v_{y}(x)$ and the local packing fraction profile $\phi(x)$ have been calculated from image analysis for four apertures $K=5,10,15$, and 20 and this for five apparent gravities $\Gamma=1,5,10$, 15, and 19.6. In the following, and for illustration purpose, we only present the different profiles for the five apparent gravities for the aperture corresponding to $40 \mathrm{~mm}(K=20)$. The analysis will be presented in two steps. First, we describe the profile and the fitting function used to phenomenologically capture the behaviour of the profiles. Second, the parameter obtained for the fitting curves are analyzed.

The figure $8 \mathrm{a}$ presents the vertical velocity $v_{y}(x)$ profiles. By construction, the vertical velocity is zero for $x / D=-D / 2 d$ and $x / D=D / 2 d$. The profiles are found to be well adjusted by

$$
v_{y}(x)=v_{y}(0) \sqrt{D^{2}-x^{2}}
$$

$v_{y}(0)$ is the maximum speed found by fitting Eq.(5) to the data [29,24]. It is important to note that this empirical profile works for all the considered apertures $K$.

The figure Fig.8b presents the local area fraction $\phi(x)$ profiles. The packing fraction is found to be around 0.40 along the border of the aperture. This value is much smaller than the value of $\phi(0)$ which is found between 0.65 and 0.75 in the center of the aperture where $\phi(x)$ is maximum. In other words, the granular packing is reduced close to the borders of the aperture. In order to observe a motion in a compact granular material, the material has to reduce locally the packing fraction. This phenomenon is better known as dilatancy process $[30,31]$. This occurs in the vicinity of the borders of the aperture while at the 


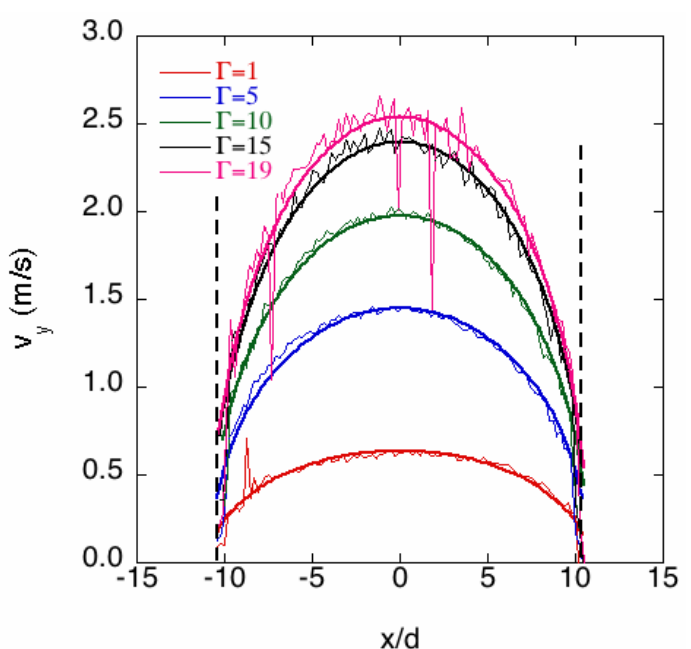

(a)

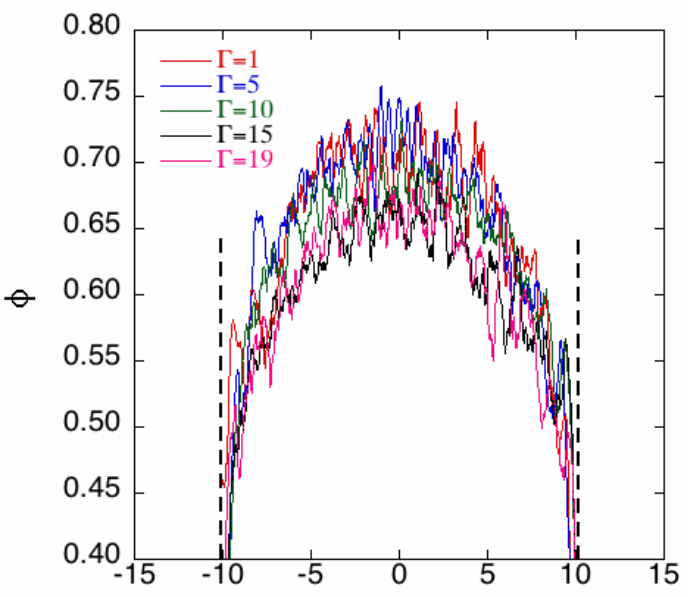

(b)

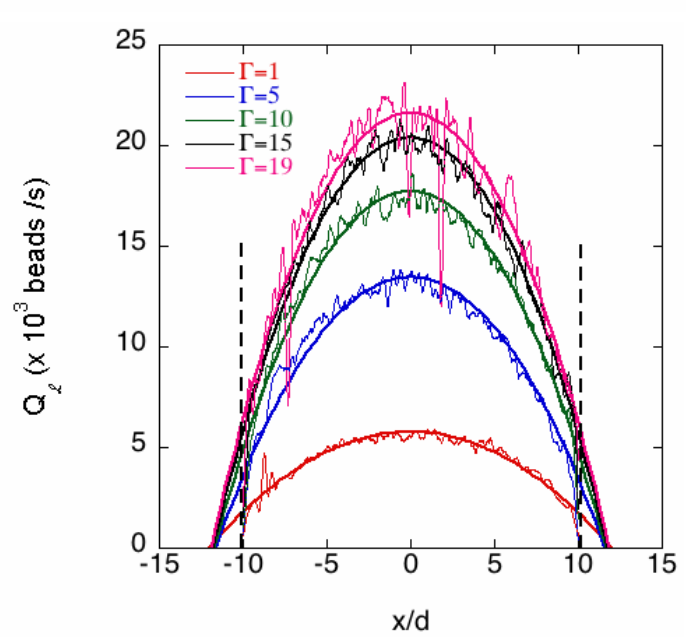

(c)

Fig. 8 (colour online) 2DProfiles $v_{y}(x), \phi(x)$ and $Q_{\ell}(x)$ are plotted for five apparent gravity, namely $\Gamma=1,5,10,15$ and 19 . Only the profiles obtained for an aperture of $40 \mathrm{~mm}(K=20)$ are presented. 
middle, a large block of granular material can flow out of the silo, conserving the initial packing fraction.

Contrary to the proposed fit for the packing fraction profile found in ref.[24], we prefer here to fit by a parabola centered at the middle of the aperture, i.e. $\phi(x)=\phi(0)\left(1+b(x / d)^{2}\right)$ where $\phi(0)$ and $b$ are fitting parameters. The parabolic law is in good agreement with the experimental data and allows to easily extract the maximum of packing fraction $\phi(0)$. The averaged values of the packing fraction $\bar{\phi}(x)$ given by

$$
\bar{\phi}=\frac{1}{d D} \int_{-D / 2}^{D / 2} \phi(0)\left(1+b(x / d)^{2}\right) d x
$$

has been computed for the different aperture sizes and for all the considered reduced gravities. The results have been replaced in Eq.(7) in order to deduce the values of $\beta$. This parameter is reported in Fig.9a as a function of the apparent gravity $\Gamma$ and for four apertures (see legend). One finds that, for large apertures $K=15$ and 20, the value of $\beta$ remains constant and close to 0.5 . That shows (i) that the fluidized area $A_{f}$ remains constant and (ii) that the characteristic free fall length corresponds to half of the aperture size as predicted by Beverloo and as observed in ref.[26]. Indeed, when the flow is jammed, the arches are found to have semi-circular shape with a radius equal to $D / 2$ which corresponds rather to $\beta=0.5$. That contrasts with the found values of $\beta$ for small apertures $(K<15)$ and large accelerations $(\Gamma>10)$. In these case, the $\beta$ parameter is larger than 0.5 and increases with the acceleration. That shows that a larger area has to be fluidized in order to ensure the flow. Finally, we compute the local flow $Q_{\ell}(x)$ (expressed in number of beads per second) which is given by

$$
Q_{\ell}(x)=4 v_{y}(x) D \phi(x) / \pi d^{2}
$$

The local flow of particles $Q_{\ell}(x)$ profiles are presented in Fig.8c. The maximum occurs at the center of the aperture and increases when the apparent gravity is increased. This observation remains consistent with the previous observations and with the Beverloo law. By construction, the local flows at $x=D / 2$ and $-D / 2$ are equal to zero as $Q_{\ell}$ results from the product of $v_{y}$ and $\phi$. However, the local flow does not goes to zero continuously. Again, to capture phenomenologically the main features, parabola profiles have been fitted. They are represented by curves in Fig.8c. Remarkably, according to the fits, the local flows vanish for $x$ values that does not seem to depend on $\Gamma$. We defined $\lambda d$ as being the value of abscissa $x$ for which $Q_{\ell}$ vanishes, i.e. $Q_{\ell}(\lambda d)=0$. The values $\lambda$ are reported in the inset of Fig.9b as a function of the apparent gravity $\Gamma$ for the four considered apertures. The horizontal lines correspond to the average values of $\lambda$. That shows that $\lambda$ is independent of $\Gamma$.

All the computed values $\lambda$ are reported as a function of the aperture $K$ for the five considered apparent gravities, namely $\Gamma=1,5,10,15$, and 19.6. The relation between the aperture and $\lambda$ is linear, namely

$$
\lambda=0.09 K
$$




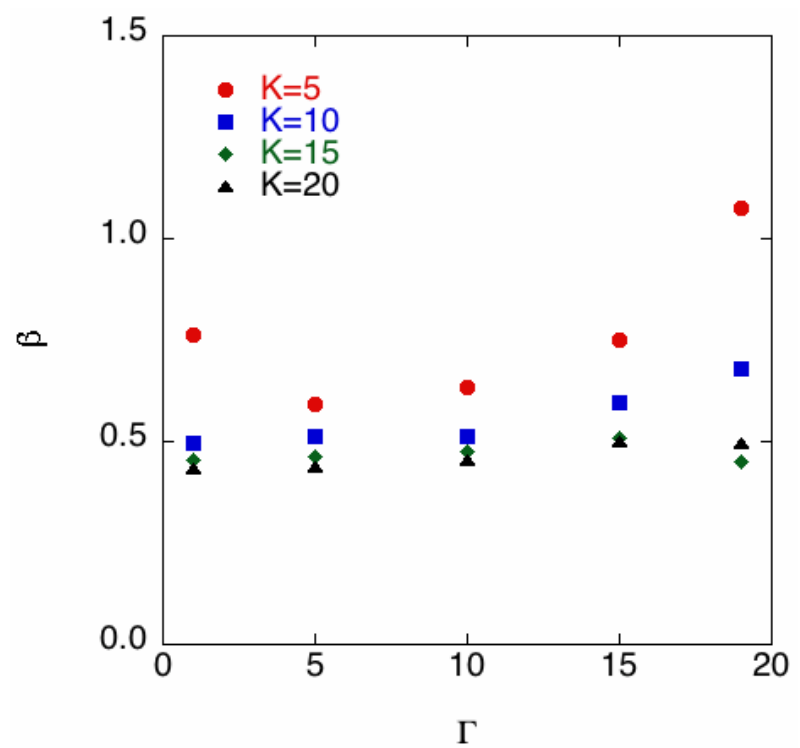

(a)

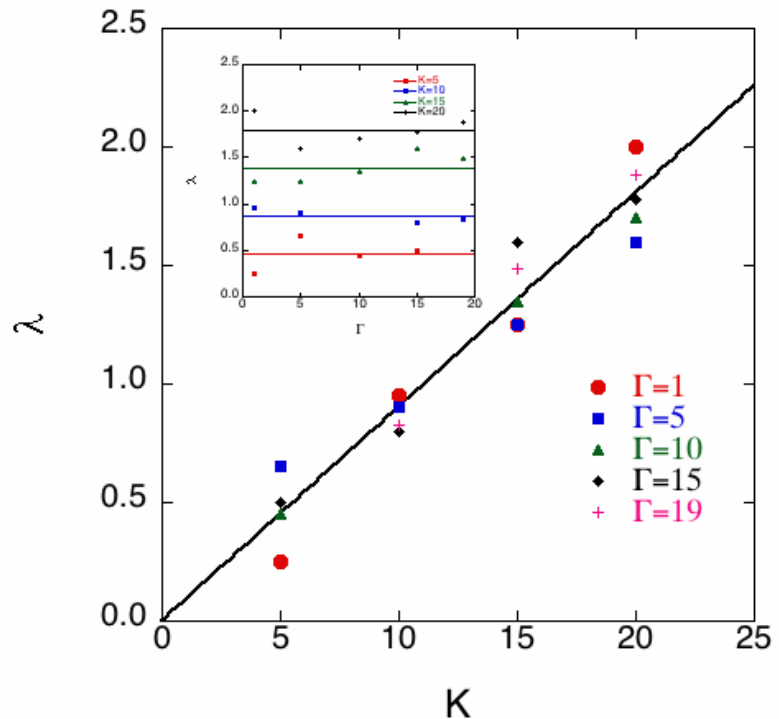

(b)

Fig. $92 \mathrm{D}$ (a) The parameter $\beta$ ( $\beta D$ represents the equivalent free fall length) is presented as a function of the reduced acceleration $\Gamma$ for four aperture sizes $K$. (b) Parameter $\lambda$ ( $\lambda d$ is the slip length of the local flow) as a function of the aperture $K$ for different reduced accelerations. In the inset, the parameter $\lambda$ is presented as a function of the apparent acceleration $\Gamma$ for the four considered aperture sizes (see legend). 


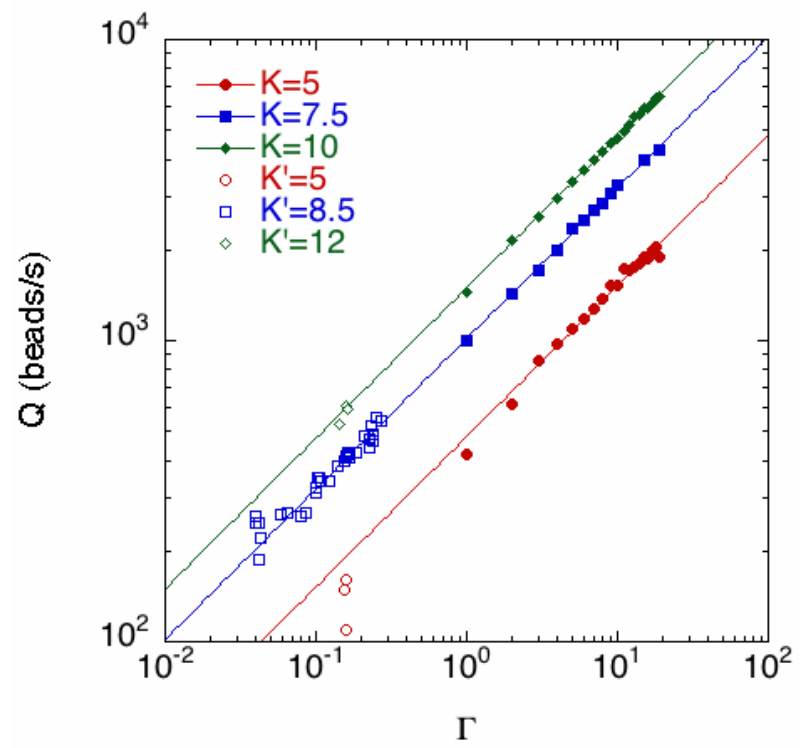

Fig. 10 2D low gravity Comparison of the flow $Q$ in high gravity conditions (LDC, plain symbols) and in low gravity condition (SDC, open symbols) as a function the apparent acceleration $\Gamma$. The plain circles, squares and diamonds were obtained for a reduced aperture $K=5,7.5$ and 10 respectively. The open circles, squares and diamonds correspond to low apparent gravity measurement for $K^{\prime}=5,8.5$ and 12 respectively. The lines correspond to square root fit on the high gravity measurements.

From an analogy to fluid mechanics, the flow slides along the border and $\lambda d$ can be interpreted as a slip length. In the present case, the slip length is found to be independent on the aperture and on the gravity. This later fact indicates that only the geometry determines the slip length $\lambda d$.

The existence of the slip length $\lambda$ should be related to internal rearrangements of the packing near the grains that flow. It supports the idea that the flow near the aperture creates defects (called spots) diffusing towards the top of the packing, providing bead motion $[32,33]$.

\subsection{Low gravity: 2D case}

Finally, we tested the silo discharge of a monolayer of beads submitted to a horizontal acceleration. As explained in the Experimental details section, especially, one aperture size was tested, i.e. $K^{\prime}=D / d=8.5$. We presented also results with $K^{\prime}=5$ and 12 for a chosen acceleration $\Gamma \approx 0.15$. The low-g measurements are reported and compared to high-g results for $K=5$, 7.5 and 10 in Fig.10. The solid lines correspond to fits of the high gravity measurements by a square root. A good agreement is found between the high and the low gravity measurements, namely the square root of the apparent 
gravity scaling works for a large range of apparent gravities. Note that the flows found under low apparent gravity conditions are lowered compared to flows obtained at high gravity characterised for same aperture sizes. In the present case, the flow found for an aperture of $K^{\prime}=8.5$ in low gravity conditions corresponds to the flow for an aperture of $K=7.5$ in high gravity experiments. This is probably due to the fact that the beads are rolling in low gravity experiments and are falling in high gravity configuration. The flows found in the low apparent gravity experiments are therefore lowered in the horizontal configuration compared to the vertical one.

\section{Conclusion}

Regarding our experimental results for 2D and for 3D conditions and for low and high apparent gravity environment, the square root dependence of the flow on the gravity can be considered as a fact. The apparent gravity does not significantly increase the minimum aperture size below which the flow is jammed. To increase the flow rate out of a silo, the silo can be centrifugated without any risk of jamming. In addition, thanks to the local properties analysis of the flow, we deduced that the fluidized area of the packing remains constant for any apparent gravity when the aperture is larger than 10 times the bead size. In this case, we found that the vertical size of the fluidized area is comparable to the half of the orifice size. On the other hand, the fluidized area increases with the acceleration for small aperture. Finally, by analyzing the local flow, a slip length has been evidenced which is found to equal $10 \%$ of the aperture size. Further experiments should be envisaged in order to determine the static behavior of the granular packing under high-gravity: gravity driven compaction, modification of the force network by gravity, angle of repose...

Acknowledgements SD and GL thanks FNRS for financial support. Part of this work was supported by Université de Liège (Credit voyage etudiant and Bourse Post-Doc). The authors would like to thank the European Space Agency for the access to the Large Diameter Centrifuge in ESTEC.

\section{References}

1. D.J. Scheeres, C.M. Hartzell, P. Sánchez, and M. Swift, Scaling forces to asteroid surfaces: The role of cohesion, Icarus 210, 968-984 (2010).

2. T. Arndt, A. Brucks, J.M. Ottino, and R.M. Lueptow, Creeping granular motion under variable gravity levels, Phys. Rev. E 74, 031307 (2006).

3. A. Brucks, T. Arndt, J.M. Ottino, and R.M. Lueptow, Behavior of flowing granular materials under variable g, Phys. Rev. E 75, 032301 (2007).

4. M.G. Kleinhans, H. Markies, S.J. de Vet, A.C. in 't Veld, and F.N. Postema, Static and dynamic angles of repose in loose granular materials under reduced gravity, J. of Geoph. Res. 116, E11004 (2011).

5. S. Dorbolo, T. Scheller, F. Ludewig, G. Lumay, and N. Vandewalle, Influence of a reduced gravity on the volume fraction of a monolayer of spherical grains, Phys. Rev. E 84, 041305 (2011)

6. http://www.youtube.com/watch? $v=$ twlSIdZufTs 
7. F. Huber-Burnand, Sur l'coulement et la pression du sable, Ann. de Chimie et de Physique 50, 159-173 (1829); Lieut. T.S. Brown, Experiments on the resistance of sand to motion through tubes, with especial reference to its use in the blasting of rocks, made at Fort Adams, Newport harbour, under the direction of Col. Totten, translated in English, F. Huber-Burnand, J. of the Franklin Inst. 22, 1-8 (1836).

8. W.A. Beverloo, H.A. Leninger, and J. van de Valde, The flow of granular solids through orifices, Chem. Eng. Sci. 15, 260-269 (1961).

9. L. Vanel, Ph. Claudin, J.-Ph. Bouchaud, M.E. Cates, E. Clément, and J.P. Wittmer, Stresses in Silos: Comparison Between Theoretical Models and New Experiments, Phys. Rev. Lett. 84, 1439-1442 (2000).

10. Y. Bertho, F. Giorgiutti-Dauphiné, and J.P. Hulin, Dynamical Janssen Effect on Granular Packing with Moving Walls, Phys. Rev. Lett. 90, 144301 (2003).

11. H. Pacheco-Martinez, H.J. van Gerner, and J.C. Ruiz-Suárez, Storage and discharge of a granular fluid, Phys. Rev. E 77, 021303 (2008).

12. B. De-Song, Z. Xun-Sheng, X. Guang-Lei, P. Zheng-Quan, T. Xiao-Wei, and L. KunQuan, Critical phenomenon of granular flow on a conveyor belt, Phys. Rev. E 67, 062301 (2003).

13. M.A. Aguirre, J.G. Grande, A. Calvo, L.A. Pugnaloni, and J.C. Géminard, Pressure Independence of Granular Flow through an Aperture, Phys. Rev. Lett. 104, 238002 (2010).

14. M.A. Aguirre, J.G. Grande, A. Calvo, L.A. Pugnaloni, and J.C. Géminard, Granular flow through an aperture: Pressure and flow rate are independent, Phys. Rev.E 83, 061305 (2011).

15. C. Perge, M.A. Aguirre, P.A. Gago, L.A. Pugnaloni, D. Le Tourneau, and J.C. Géminard, Evolution of pressure profiles during the discharge of a silo, Phys. Rev. E 85, 021303 (2012).

16. I. Zuriguel, L.A. Pugnaloni, A. Garcimartín, and D. Maza, Jamming during the discharge of grains from a silo described as a percolating transition, Phys. Rev. E 68, 030301 (R) (2003).

17. C. Mankoc, A. Janda, R. Arévalo, J.M. Pastor, I. Zuriguel, A. Garcimartín, and D. Maza, The flow rate of granular materials through an orifice, Gran. Matter 9, 407-414 (2007).

18. A. Janda, I. Zuriguel, A. Garcimartín, L. A. Pugnaloni and D. Maza, Jamming and critical outlet size in the discharge of a two-dimensional silo, Europhys. Lett. 84, 44002 (2008).

19. G. Lumay and N. Vandewalle, Controlled flow of smart powders, Phys. Rev. E 78, 061302 (2008).

20. E. Mersch, G. Lumay, F. Boschini, and N. Vandewalle, Effect of an electric field on an intermittent granular flow, Phys. Rev. E 81, 041309 (2010).

21. Y. Bertho, C. Becco, and N. Vandewalle, Dense bubble flow in a silo: An unusual flow of a dispersed medium, Phys. Rev. E 73, 056309 (2006).

22. A. Janda, D. Maza, A. Garcimartín, E. Kolb, J. Lanuza, and E. Clément, Unjamming a granular hopper by vibration, Europhys. Lett. 87, 24002 (2009).

23. I. Zuriguel, A. Janda, A. Garcimartín, C. Lozano, R. Arévalo, and Diego Maza, Silo Clogging Reduction by the Presence of an Obstacle, Phys. Rev. Lett. 107, 278001 (2011).

24. A. Janda, I. Zuriguel, and D. Maza, Flow Rate of Particles through Apertures Obtained from Self-Similar Density and Velocity Profiles, Phys. Rev. Lett. 108, 248001 (2012).

25. A. Garcimartín, I. Zuriguel, L.A. Pugnaloni and A. Janda, Shape of jamming arches in two-dimensional deposits of granular materials, Phys. Rev. E 82, 031306 (2010).

26. C. Lozano, G. Lumay, I. Zuriguel, R. C. Hidalgo, and A. Garcimartín, Breaking Arches with Vibrations: The Role of Defects, Phys. Rev. Lett. 109, 068001 (2012).

27. SiLibeads, Sigmund-Lindner, Type S, roundness $>0.89 \%$.

28. ZEUGMA, Tecnologia de Sistemas Inudstriais, Lda, large Diameter Centrifuge vibrations measurement and analysis (97278001B1), Aug. 2011.

29. A. Janda, R. Harich, I. Zuriguel, D. Maza, P. Cixous, and A. Garcimartín, Flow-rate fluctuations in the outpouring of grains from a two-dimensional silo, Phys. Rev. E $\mathbf{7 9}$ 031302 (2009). 
30. GDR Midi, On dense granular flows, Europhys. J. E 14, 341-365 (2004).

31. K. Sakaie, D. Fenistein, T.J. Carroll, M. Van Hecke, P. Umbanhowar, MR imaging of Reynolds dilatancy in the bulk of smooth granular flows, Europhys. Lett. 84, 49902 (2008)

32. M. Z. Bazant, The Spot Model for random-packing dynamics, Mechanics of Materials 38, 717-731 (2006).

33. C. H. Rycroft, M. Z. Bazant, J. Landry, and G. S. Grest, Dynamics of random packings in granular flow, Physical Review E 73, 051306 (2006). 